\title{
Socially Induced False Memories in the Absence of Misinformation
}

Ullrich Wagner ( $\nabla$ ullrich.wagner@uni-muenster.de )

University of Münster

Gerald Echterhoff

University of Münster

\section{Research Article}

Keywords: false memories, social influence, co-monitoring, joint encoding, DRM paradigm

Posted Date: September 7th, 2021

DOI: https://doi.org/10.21203/rs.3.rs-847613/v1

License: (c) (i) This work is licensed under a Creative Commons Attribution 4.0 International License. Read Full License 
Socially induced false memories in the absence of misinformation

\section{Ullrich Wagner* \& Gerald Echterhoff}

Department of Psychology, University of Münster, Germany

* Corresponding author. Contact information: Ullrich Wagner, Department of

Psychology, University of Münster, Fliednerstr. 21, 48149 Münster, Germany; Phone:

+49-251-83-39197; E-mail: ullrich.wagner@uni-muenster.de 


\section{Abstract}

Ample evidence shows that post-encoding misinformation from others can induce false memories. Here, we demonstrate in two experiments a new, tacit form of socially generated false memories, resulting from interpersonal co-monitoring at encoding without communication of misinformation. Pairs of participants jointly viewed semantically coherent word lists, presented successively in blue, green, or red letters. Each individual was instructed to memorize words presented in one of the colors. One color remained unassigned (control condition). Participants reported more false memories for non-presented words (lures) semantically related to partner-assigned than to control lists, although both list types were equally irrelevant to their own task. Notably, this effect also persisted for particularly rich memories. These findings show for the first time that social induction of false memories, even subjectively rich ones, does not necessarily require communication of deceptive information. This has important implications both theoretically and practically (e.g. in forensic contexts). 
Socially induced false memories in the absence of misinformation

Human memory is highly susceptible to social influence. Given that humans are members of an "ultrasocial” species (Tomasello, 2014), our memory contents are constantly shaped by social interaction and communication (Hirst \& Echterhoff, 2012; Rajaram \& Pereira-Pasarin, 2010). However, such influences not always strengthen veridical memories, but can also be sources of false memories, i.e., memories for events that did not actually happen (Loftus, 1997; Nash \& Ost, 2017), exemplified by the famous misinformation effect (Loftus, 2005), memory conformity (Wright et al., 2000) and social contagion of memory (Roediger, et al., 2001). A chief tool for exerting social influence are suggestive techniques, which can create even rich and vivid false memories (e.g., Loftus \& Pickrell, 1995; Shaw \& Porter, 2015). Such influence can have serious, unwanted consequences, such as biased assessments of past events, suboptimal decision-making, and unfair judgments of actors, even the imprisonment of innocent people (Bernstein \& Loftus, 2009a; Murty et al., 2016; Loftus, 2013, 2018).

Notably, these previous demonstrations of socially induced false memories rely on communication of misinformation presented after the initial encoding of relevant episodes. Participants are likely to incorporate post-encoding misinformation communicated by a social source if they assume that the source is sufficiently trustworthy (Blank, 1998; Echterhoff et al., 2005). However, more subtle mechanisms of social appraisal and perceived relevance, operating already at encoding, may likewise contribute to the creation of false memories.

In the present paper, we investigated whether false memories can be socially induced in the absence of misinformation. Research on simultaneous co-attention with others (Shteynberg, 2015), co-representation of information (see Smith \& Mackie, 2015), and distributed task performance (Eskenazi et al., 2013; Wagner et al., 2017) suggests that people encode and incorporate to a greater extent information that is relevant to (vs. not relevant to) 
interaction partners. We argue that people spontaneously monitor current stimuli for relevance to their interaction partner. Stimuli that are relevant to the partner receive more attention, are encoded more deeply, and are thus more readily accessible for further potential co-activities with their partners (see Shteynberg, 2015). As such, information processed by one's partner is a component, or building block, of shared reality created by interaction partners (Echterhoff \& Higgins, 2017)

Imagine, for instance, that upon a delivery of goods arriving at a supermarket, Billie and a colleague distribute the task of checking the labels on the unloaded packages. Billie attends to vegetables (e.g., tomatoes, onions, broccoli); the colleague attends to fruit items (e.g., pears, oranges, grapes). According to our proposal, Billie would monitor the labels not only for vegetable items but also, to some extent, for the partner's fruit items. This comonitoring would enhance the cognitive accessibility and recall not only of Billie's own items but also of the partners' items. This memory advantage for functional to the extent that it can help to regulate future co-activities and communication, such as organizing product placement and use of shelf space (Smith \& Mackie, 2016).

Now assume that apples were not delivered. In the absence of the colleague, Billie is later asked by a manager whether apples were received. We propose that, as a result of spontaneous co-processing of the partner's fruit items, Billie may falsely remember apples as having been delivered. Such false memory is socially rooted to the extent that it emerges from the spontaneous co-processing of the partner's items. However, it emerges without misinformation conveyed by the colleague.

To investigate this subtle, tacit from of false memory, we created a socially contextualized version of the well-known Deese-Roediger-McDermott (DRM) paradigm (Deese, 1959; Roediger \& McDermott, 1995). In individuals, the DRM procedure reliably induces false memories for words that are strongly associated with actually presented word lists. For example, when participants are asked to encode a word list like "table", "sit", "seat", 
.... they subsequently often remember having seen the non-presented lure "chair.". To embed this procedure into a social setting, we presented the lists within a joint encoding paradigm (Eskenazi et al., 2013). In this paradigm, two participants simultaneously watch words shown successively on a screen. Each person has to attend and react to a different subset of items (e.g., animal vs. fruit). A third subset of words (e.g., household objects) is assigned to neither participant.

Evidence shows that partner-assigned words (e.g., fruit, in our example) are later better remembered than non-assigned words (household objects), although both categories were not assigned to oneself (Eskenazi et al., 2013; Wagner et al., 2017). These results support the notion that participants co-monitored stimuli for partner-relevance, without intentional influence from another person (Smith \& Mackie, 2015; Shteynberg, 2015). What remains unknown so far, however, is whether the same social co-monitoring mechanism can also induce false memories.

The DRM paradigm is particularly apt to address this question because it reliably creates false memories. These false memories can be even subjectively rich, encompassing vivid memory details (Gallo, 2010). Richness is an important cue for evaluating the truthfulness of reported memories (Hershkowitz et al., 1997; Bernstein \& Loftus, 2009b). Apart from examining effects on overall memory, we therefore also assessed effects specifically on those memories associated with particularly rich subjective memory experiences.

We performed two experiments that applied the DRM paradigm within adapted versions of the joint encoding paradigm (Eskenzi et al., 2013). In Experiment 1, social comonitoring was explicitly instructed for each stimulus, in Experiment 2 there was no such instruction. To assess the phenomenological richness of memories, we included „Remember/Know“ decisions regarding subjective memory experience (Tulving, 1985) in Experiment 1 and asked for source memory as a specific additional memory for encoding 
context in Experiment 2. Both experiments revealed that false memories were augmented for stimuli that were relevant (vs. not relevant) to the partner, and this effect persisted for particularly rich memory experiences.

\section{Experiment 1}

In our first experiment, participants processed the social relevance of each presented stimulus explicitly during the joint encoding task, i.e., they had to decide whether the stimulus was relevant to the partner's task or not. Because pilot tests produced low false memory rates for non-self-assigned categories in a free recall format, we used a recognition test. Still, following the original DRM procedure (Roediger \& McDermott, 1995), we also included a free recall test preceding the recognition memory test. This also allowed us to test the replicability of the originally reported joint encoding effect for veridical memory, which is likewise based on free recall (Eskenazi et al, 2013; Wagner et al., 2017).

\section{Methods}

\section{Participants}

Fifty-four participants (age: $M=24.54$ years, $S D=6.58 ; 45$ female) were recruited online or personally at the University of Münster (Germany). The sample size was determined on the basis of an a-priori power analysis performed by G*Power (Faul et al., 2007) to detect an effect size $\left(d_{z}\right)$ of at least 0.5 with high statistical power $($ power $=.95$; alpha $=.05$, two-tailed). Participants took part in the study for curricular credit or the chance of winning a voucher for an online shop $(€ 10)$. The study was approved by the ethics committee of the Department of Psychology at the University of Münster and was carried out in accordance with the provisions of the Declaration of Helsinki. All participants gave informed written consent before participation.

\section{Materials and Procedure}


The experimental procedure basically followed the joint encoding procedure as described in Eskenazi et al. (2013), but with some modifications to allow the combination with the DRM paradigm. The primary modification was that not word meaning, but word color determined task relevance of a word. Participants took part in the experiment in pairs. The two participants in a pair performed the experiment at the same computer and were asked to sit comfortably next to each other, looking at the same screen. The study was introduced to them as a "word processing task with another participant." Written instructions explained the details of the task. Participants were informed that single words would be presented successively on the computer screen, and that each word could be written in one of the colors blue, green, or red.

Each participant was assigned to one of the three colors and informed that his/her task was to specifically process words that were presented in this assigned color. The two participants in each pair were always assigned to two different colors. Both of them knew from the beginning which color was assigned to the other participant and which color was assigned to nobody. The assignment of specific colors to conditions was balanced across participants. Participants were instructed to indicate for each word by a keypress whether it was assigned to themselves, to the partner, or to nobody, and if it was assigned to themselves, to try to memorize it. The pre-defined keys to perform the word categorization were "x", "c", and " $\mathrm{v}$ " (on the left part of the keyboard) for the person sitting on the left, and "1", " 2 ", and " 3 " on the numlock pad (on the right of the keyboard) for the person sitting on the right.

The word categorization task was designed to require a keypress for all stimuli (not only for self-assigned stimuli) in order to avoid a confound by perceptual cues due to audible clicks generated by the partner's keypresses (see also Wagner et al., 2017). In the critical comparison between partner-assigned and non-assigned words, such sounds would occur only in the former but not in the latter case, if instructions asked for a keypress only for selfassigned words. 
The material used in the joint encoding task consisted of 195 words from 15 German DRM word lists used by Diekelmann and colleagues (2008). Each list was composed of 13 words (we shortened the original list length of 15 by two words), which were semantically associated with a non-presented theme word, serving as the "critical lure". For instance, the list for the critical lure music contained note, sound, piano, sing, band, melody, guitar, concert, instrument, symphony, jazz, orchestra, and art. Within a list, these words were ordered according to their strength of association with the critical lure. That is, the word in the first position (here: note) had the highest associative strength in relation to the critical lure (here: music), the last position (here: art) had the weakest associative strength to it. (We shortened the original list length in Diekelmann et al., 2008, from 15 to 13, leaving out words from the original positions 5 and 15 of each list.). Importantly, each list was assigned to one of three colors (blue, green, and red) and was presented with a corresponding color. Accordingly, five lists were shown in red, green, and blue color, respectively. In the recognition memory test, additional words not presented at encoding were included. Fifteen of these new words were the critical lures of the previously presented DRM lists. Additional 42 words were distractors (words from the original positions 5 and 15 and twelve completely unrelated words).

E-Prime (Version 2.0) was used to run the experiment. Two practice runs (each containing nine words not included in the main run) were performed to familiarize participants with the task. The main run included all 15 word lists (five for each category), presented listwise in a pseudo-random order in such a way that a word list with a specific task assignment was never directly followed or preceded by another word list with the same task assignment. Additional nine words (three of each color) served as distractors, with three of them presented in the beginning (as primacy buffer) and three in the end (as recency buffer). All words were presented for 2,500 ms one after another in the middle of the screen and appeared in their specified color (blue, green, or red) on a black background. Regardless of 
the response time, the words remained visible for the whole 2,500 ms. Participants were instructed in advance to be focused on the task across the whole run and not to talk. Instructions also pointed out that response times did not matter, so that it was not important to do the keypresses for the word categorizations faster than the partner.

After having finished the task, the two participants were separated to perform the subsequent memory tests individually. First, a free recall test was performed, for which each participant received an empty sheet of paper. Unexpectedly, they were asked to write down as many words as possible not only from the self-assigned category in the previous task, but from all three categories. Then, the recognition memory test followed on a computer. Altogether 132 words, written in white color on a black background, were presented successively in random order. Seventy-five of the words had been actually presented during the word categorization task (items from positions 1, 4, 5, 12, and 13 of each list). Additional 57 words were new. Fifteen of these new words were the critical lures of the previously presented DRM lists, which are in the focus of interest here.

The remaining new words were distractors (words from the original positions 5 and 15 and twelve additional unrelated words). For each word, participants had to indicate whether it had been presented during the word categorization task or not, whether their answer was based on a "remember" or "know" experience (Tulving, 1985). They were instructed to give a "remember" answer if they were able to retrieve additional details or context information from the respective encoding situation, and to give a "know" answer if this was not the case. Participants completed the task at their own pace.

\section{Statistical Analyses}

Free recall data were only analyzed with respect to veridical memory to test the replicability of previous free recall findings (Eskenazi et al, 2013; Wagner et al., 2017) with this modification of the same paradigm. The dependent variable was the percentage of recalled words from that had been actually presented in each word category (self, other, 
none). As anticipated, the percentage of false memories (recalled critical lures) was too low in the non-self-assigned word categories to allow useful statistical comparisons in this memory test $(<5 \%)$.

Dependent variables in the recognition memory test were the number of "Yes" answers to the question whether the presented item was shown during the word categorization task or not (overall memory). For an additional analysis on only memories with the richest subjective memory experiences, only "Yes" answers with additional indication of a "remember" experience were considered. For false memories, the percentage of respective answers was calculated with regard to the number of critical lures in each word category (self, other, none), and for veridical memory with regard to the list words that had actually been presented.

All analyses were based on one-way analyses of variance (ANOVA) with the withinsubject factor word category (self, other, none), followed by pairwise t-test comparisons. The critical pairwise comparison of interest was between the two categories "other" and "none". Percentages for self-assigned words were expected to be generally higher than those in the corresponding "other" and "none" categories.

\section{Results}

\section{Free Recall}

There was a significant effect of word category $\left(F(2,106)=255.79, p<.001, \eta_{p}{ }^{2}=.828\right)$. Recall for self-assigned words $(M=70.52 \%)$ was substantially higher than for other-assigned $(M=8.04 \%)$ and non-assigned words $(M=5.11 \%), p s<.001$. Recall for other-assigned word was also significantly higher than for non-assigned words $(p<.05)$. Thus, free recall data on veridical memory replicated previous free recall findings obtained in the same experimental paradigm with different material (Eskenazi et al, 2013; Wagner et al., 2017). As noted, false recall of critical lures from the non-self-assigned word lists were at floor level, with many 
participants writing down no critical lures at all. False recall of critical lures from the selfassigned word category was $27 \%$ on average.

\section{Recognition Memory}

Data from the recognition memory test are shown in Table 1. For both veridical and false memories, a significant effect of word category was obtained when overall memory was assessed $\left(F(2,106)=213,18, p<.001, \eta_{p}^{2}=.801\right.$, and $F(2,106)=44.06, p<.001, \eta_{p}{ }^{2}=.454$, respectively) and also when only „remember“ answers were considered $(F(2,106)=239.28, p$ $<.001, \eta_{p}^{2}=.819$, and $F(2,106)=59.66, p<.001, \eta_{p}^{2}=.530$, respectively). In all cases, the numbers were substantially higher for self-assigned words than for words from the other two categories (all $p s<.001)$. Critically, there were also substantially more false memories in the other-assigned than in the non-assigned word category, both in the overall memory analysis and when only ,remember“ answers were taken into account ( $p$ s $<.05$; Figure 1$)$. For overall memory, but not for specifically „remember“-based memory, this was also accompanied by significantly stronger veridical memory for other-assigned than for non-assigned words ( $p$ $<.05)$.

\section{Experiment 2}

Experiment 2 replicated and extended Experiment 1. Importantly, we tested if the effect would persist in the absence of explicit instructions to process the social meaning of stimuli during the joint-encoding task. For this purpose, participants were not asked to determine word assignment (self, other, or none) in the categorization task, but to indicate the word color (blue, green, or red). Thus, participants had to infer the differential social meaning of the non-self-stimuli on their own. If the effect persists under these conditions, this would further support the notion that the underlying process of social appraisal occurs spontaneously 
(Smith \& Mackie, 2015; Shteynberg, 2015). In addition, two procedural changes were introduced in Experiment 2. First, the initial free recall test was omitted. Second, we used another method to assess particularly rich memories. For each word that participants recognized as having been presented, they were not asked for their „remember“ vs. „know“ experience, but to indicate to which of the three word categories the recognized word belonged (source memory).

\section{Methods}

\section{Participants}

Sixty participants (age: $M=24.91$ years, $S D=3.84 ; 42$ female) were recruited online or personally at the University of Münster. Participants took part in the study for curricular credit or the chance of winning a voucher for an online shop $(€ 10)$. All participants gave informed written consent before participation. One participant was excluded from statistical analyses due to an extremely liberal response bias, as indicated by a percentage of "Yes" answers $>95 \%$ even for the distractors in the recognition memory test that were not critical lures.

\section{Materials and Procedure}

Material and procedures were identical to Experiment 1, with the following exceptions: First, in the word categorization task, participants were asked to determine the color of the presented word (not the word assignment). i.e., whether it was written in blue, green, or red color. Second, there was no free recall test, but only a recognition memory test. Third, in the recognition test, participants were specifically asked for their source memory, as an alternative to the "remember/know" distinction to define rich memories. Specifically, for "Yes" answer in the recognition test, participants were not asked to report their subjective experience of "remembering" vs. "knowing." Instead, they indicated the word category of the recognized word, i.e., self-assigned, other-assigned, non-assigned word category. We added 
these source-memory responses to tap into participants' perception of the stimuli's social relevance.

\section{Results}

Data for the recognition memory test (Table 2) replicate the findings from Experiment 1. For both veridical and false memories, a significant effect of word category was obtained when overall memory was assessed $\left(F(2,118)=114.00, \mathrm{p}<.001, \eta_{p}{ }^{2}=.659\right.$, and $\mathrm{F}(2,118)=$ $44.06, p<.001, \eta_{p}^{2}=.454$, respectively) and also when only memories with additional corresponding source memory were considered $\left(F(2,118)=95.63, p<.001, \eta_{p}^{2}=.618\right.$, and $F(2,118)=56.38, p<.001, \eta_{p}^{2}=.489$, respectively $)$. The percentages were again in all cases substantially higher for self-assigned words than for words from the other two categories (all $p s<.001)$. Critically, there were again also substantially more false memories in the otherassigned than in the non-assigned word category, both in the overall memory analysis ( $p$ $<.05)$ and when only memories with additionally remembered source were taken into account $(p<.01$; Figure 1). For overall memory, but not for specifically source-associated memory, there was also significantly stronger veridical memory for other-assigned than for nonassigned words $(p<.05)$.

\section{General Discussion}

The present study reveals a novel type of socially driven false memories, which emerges from co-processing during initial encoding. In contrast to previous demonstrations, this effect appears to emerge unobtrusively from the spontaneous monitoring of socially relevant stimuli at encoding rather than by post-encoding presentation of misinformation leading to conflicting memory representations. Using the DRM false memory task (Roediger \& McDermott, 1995) within the social context of a distributed task sharing paradigm (Eskenazi et al, 2013), we replicated previous findings that the mere knowledge that words 
were specifically relevant to a partner's task led to enhanced memory for these words (Eskenazi et al, 2013; Wagner et al., 2017). Critically, however, false memories for words that were semantically highly associated with the actually presented partner-relevant words were also substantially enhanced. As shown in Experiment 2, this was also the case when instructions did not directly demand processing of social relevance vs. irrelevance of each stimulus during task performance (as in Experiment 1). This is in line with the assumption of naturally occurring involuntary social appraisal as the underlying mechanism (Smith \& Mackie, 2015; Shteynberg, 2015).

Although the demonstrated social process of false-memory formation is new, the basic cognitive mechanism that it affects is not. It is the same mechanism of associative semantic inference already observed in many previous studies performed with individual participants, where DRM lists inherently only have self-relevance (Gallo, 2010). This basic effect was clearly confirmed here for the self-assigned words that showed in all analyses the strongest extent of false memory generation (in parallel to the best veridical memory for actually presented words from this word category). The strongest effect for the self-assigned words is not surprising because participants had to focus their attention to these words already by instruction, just as in classical DRM studies.

Critically, the present data show, for the first time, that the same cognitive mechanism is effective, albeit in an attenuated manner, when attention to stimuli is solely driven by attribution of social relevance. Participants developed more false memories for word lists that were relevant to the partner than for word lists that were not, although both types of word lists were equally irrelevant to perform the own task and were therefore only encoded incidentally. Compared to paradigms relying on post-event misinformation (Wright et al., 2000; Roediger et al., 2001; Loftus, 2005), social influence here is very subtle, being exerted only passively by an interaction partner. Nevertheless, a remarkable feature of the effects in the present experiments is that the false memories that they create are relatively rich and vivid, 
accompanied by additional memories for detail or context. Notably, when only these memories with high subjective richness were considered, the same statistical pattern was obtained.

False memories from interpersonal co-monitoring at encoding also differ from other paradigms that describe negative effects of social processes on memory performance, such as "collaborative inhibition" (Rajaram \& Pereira-Pasarin, 2010) and "socially shared retrievalinduced forgetting" (Cuc et al., 2007). These phenomena investigate the extent of forgetting of originally encoded material, rather than the active creation of false memories, i.e., of nonveridical new memories. Furthermore, like misinformation studies, they are not interested in social influence at encoding, but on effects of social processes after encoding. This latter aspect also distinguishes the present effects from false memories of action performance from the mere observation of others' actions (Lindner et al., 2010), and from effects of collaborative recall of DRM lists on the transmission of false memories (Maswood \& Rajaram, 2019).

The present findings not only advance our theoretical understanding of how social and cognitive factors interact in false memory formation. Because episodic memory also affects the quality of judgment and decision-making (Del Missier et al., 2013; Murty et al., 2016), this understanding is also relevant to applied research, e.g. in the context of economic and consumer choices (Alba et al., 1991; Bernstein \& Loftus, 2009a). Particularly obvious is the pertinence to the forensic domain, considering the fact that a substantial proportion of false legal convictions is based on false eyewitness memories (Rattner, 1988; Loftus, 2013). Notably, research on social factors in false memory generation has so far mainly focused on interpersonal communication in active social interaction taking place after encoding. Our results show that it is likewise important to consider social determinants that exert their influence more unobtrusively already at encoding. 
In sum, our data show for the first time that social induction of false memories, notably even subjectively rich ones, does not necessarily require communication of deceptive information. Rather than acting through post-encoding open communication, the mechanism of co-monitoring that we describe here is driven by socially guided attention at encoding. Because social guidance of attention is ubiquitous in human life (Tomasello, 2014; Shteynberg, 2015; Smith \& Mackie, 2015), this potential source of even rich false memories deserves deeper consideration in future research, including applied research (e.g., in the forensic domain).

\section{References}

Alba, J.W., Hutchinson, J.W., \& Lynch, J.G. (1991). Memory and Decision Making. In T.S. Robertson \& H.H. Kassarjian (Eds.): Handbook of Consumer Behavior. Englewood Cliffs, NJ: Prentice Hall (pp. 1-49).

Bernstein, D.M., \& Loftus, E.F. (2009a). The consequences of false memories for food preferences and choices. Perspectives on Psychological Science, 4(4), 135-139. https://doi.org/10.1111/j.1745-6924.2009.01113.x

Bernstein, D.M., \& Loftus, E.F. (2009b). How to tell if a particular memory is true or false. Perspectives on Psychological Science, 4(4), 370-374. https://doi.org/10.1111/j.17456924.2009.01140.x

Blank, H. (1998). Memory states and memory tasks: An integrative framework for eyewitness memory and suggestibility. Memory, 6(5), 481-529. https://doi.org/10.1080/741943086

Cuc, A., Koppel, J., \& Hirst, W. (2007). Silence is not golden: A case for socially shared retrieval-induced forgetting. Psychological Science, 18(8), 727-733. https://doi.org/10.1111/j.1467-9280.2007.01967.x

Deese, J. (1959). On the prediction of occurrence of particular verbal intrusions in immediate recall. Journal of Experimental Psychology, 58(1), 17-22. https://doi.org/10.1037/h0046671 
Del Missier, F., Mäntylä, T., Hannson, P., Bruine de Bruin, W., Parker, A.M., \& Nilsson, L.G. (2013). The multifold relationship between memory and decision-making: An individual-differences approach. Journal of Experimental Psychology: Learning, Memory, and Cognition, 39(4), 1344-1364. https://doi.org/10.1037/e519682015-035

Echterhoff, E., \& Higgins, E.T. (2017). Creating shared reality in interpersonal and intergroup communication: The role of epistemic processes and their interplay. European Review of Social Psychology, 28(1), 175-226.

https://doi.org/10.1080/10463283.2017.1333315

Echterhoff, E., \& Hirst, W., \& Hussy, W. (2005). How eyewitnesses resist misinformation: Social postwarnings and the monitoring of memory characteristics. Memory \& Cognition, 33, 220-282. https://doi.org/10.3758/bf03193073

Eskenazi, T., Doerrfeld, A., Logan, G. D., Knoblich, G., \& Sebanz, N. (2013). Your words are my words: Effects of acting together on encoding. Quarterly Journal of Experimental Psychology, 66(5), 1026-1034. https://doi.org/10.1080/17470218.2012.725058

Diekelmann S, Landolt H-P, Lahl O, Born J, \& Wagner U. (2008) Sleep loss produces false memories. PLoS ONE 3(10): e3512. https://doi.org/10.1371/ journal.pone.0003512

Faul, F. Erdfelder, E., Lang, A.-G. \& Buchner, A. (2007). G*Power 3: A flexible statistical power-analysis program for the social, behavioral, and biomedical sciences. Behavior Research Methods, 39, 175-191. https://doi.org/10.3758/BF03193146

Gallo, D. A. (2010). False memories and fantastic beliefs: 15 years of the DRM illusion. Memory \& Cognition, 38(7), 833-848. https://doi.org/10.3758/mc.38.7.833

Hershkowitz, I., Lamb, M.E., Sternberg, K.J. \& Espin, P.W. (1997). The relationships among interviewer utterance type, CBCA scores and the richness of children's responses. Legal and Criminal Psychology, 2, 169-176. https://doi.org/10.1111/j.20448333.1997.tb00341.x

Hirst, W., \& Echterhoff, G. (2012). Remembering in conversations: The social sharing and reshaping of memories. Annual Review of Psychology, 63(1), 55-79. https://doi.org/10.1146/annurev-psych-120710-100340 
Lindner, I., Echterhoff, G., Davidson, P. S., \& Brand, M. (2010). Observation inflation: Your actions become mine. Psychological Science, 21(9), 1291-1299. https://doi.org/10.1177/0956797610379860

Loftus, E. F. (1997). Memory for a past that never was. Current Directions in Psychological Science, 6(3), 60-65. https://doi.org/10.1111/1467-8721.ep11512654

Loftus, E. F. (2005). Planting misinformation in the human mind: A 30-year investigation of the malleability of memory. Learning \& Memory, 12(4), 361-366. https://doi.org/10.1101/lm.94705

Loftus, E. F. (2013). 25 years of eyewitness science......finally pays off. Perspectives on Psychological Science, 8(5), 556-557. https://doi.org/10.1177/1745691613500995

Loftus, E. F. (2018). Eyewitness science and the legal system. Annual Review of Law and Social Science, 14(1), 1-10. https://doi.org/10.1146/annurev-lawsocsci-101317030850

Loftus, E. F., \& Pickrell, J. E. (1995). The formation of false memories. Psychiatric Annals, 25(12), 720-725. https://doi.org/10.3928/0048-5713-19951201-07

Maswood, R,, \& Rajaram, S. (2019). Social transmission of false memory in small groups and large networks. Topics in Cognitive Science, 11, 687-709. https://doi.org/10.1111/tops.12348

Murty, V.P., FeldmannHall, O., Hunter, L.E., Phelps, E.A., \& Davachi, L. (2016). Episodic memories predict adaptive value-based decision-making. Journal of Experimental Psychology: General, 145(5), 548-558. https://doi.org/10.1037/xge0000158

Nash, R. A., \& Ost, J. (2016). False and Distorted Memories. London / New York: Routledge. https://doi.org/10.4324/9781315736242

Rajaram, S., \& Pereira-Pasarin, L. P. (2010). Collaborative memory: Cognitive research and theory. Perspectives on Psychological Science, 5(6), 649-663. https://doi.org/10.1177/1745691610388763 
Rattner, A. (1988). Convicted but innocent: Wrongful conviction and the criminal justice system. Law and Human Behavior, 12(3), 283-293.

https://doi.org/10.1007/bf01044385

Roediger, H. L., \& McDermott, K. B. (1995). Creating false memories: Remembering words not presented in lists. Journal of Experimental Psychology: Learning, Memory, and Cognition, 21(4), 803-814. https://doi.org/10.1037/0278-7393.21.4.803

Roediger, H. L., Meade, M. L., \& Bergman, E. T. (2001). Social contagion of memory. Psychonomic Bulletin \& Review, 8(2), 365-371. https://doi.org/10.3758/bf03196174

Shaw, J., \& Porter, S. (2015). Constructing rich false memories of committing crime. Psychological Science, 26(3), 291-301. https://doi.org/10.1177/0956797614562862

Shteynberg, G. (2015). Shared attention. Perspectives on Psychological Science, 10(5), 579590. https://doi.org/10.1177/1745691615589104

Smith, E. R., \& Mackie, D. M. (2015). Representation and incorporation of close others' responses: The RICOR model of social influence. Personality and Social Psychology Review, 20(4), 311-331. https://doi.org/10.1177/1088868315598256

Smith, E. R., \& Mackie, D. M. (2016). Influence from representations of others' responses: social priming meets social influence. Current Opinion in Psychology, 12, 22-25. http://dx.doi.org/10.1016/j.copsyc.2016.04.012

Tomasello, M. (2014). The ultra-social animal. European Journal of Social Psychology, 44(3), 187-194. https://doi.org/10.1002/ejsp.2015

Tulving, E. (1985). Memory and consciousness. Canadian Psychology/Psychologie Canadienne, 26(1), 1-12. https://doi.org/10.1037/h0080017

Wagner, U., Giesen, A. , Knausenberger, J., \& Echterhoff, G. (2017). The joint action effect on memory as a social phenomenon: The role of cued attention and psychological distance. Frontiers in Psychology, 8, 1697. https://doi.org/10.3389/fpsyg.2017.01697

Wright, D. B., Self, G., \& Justice, C. (2000). Memory conformity: Exploring misinformation effects when presented by another person. British Journal of Psychology, 91(2), 189202. https://doi.org/10.1348/000712600161781 
Table 1. Veridical and false memories in Experiment 1

\begin{tabular}{|c|c|c|c|c|c|c|c|c|}
\hline & \multicolumn{4}{|c|}{ Overall memory } & \multicolumn{4}{|c|}{ "Remember" memory } \\
\hline & \multirow{2}{*}{\multicolumn{2}{|c|}{$\begin{array}{l}\text { Veridical } \\
\text { memories }\end{array}$}} & \multirow{2}{*}{\multicolumn{2}{|c|}{$\begin{array}{c}\text { False } \\
\text { memories }\end{array}$}} & \multirow{2}{*}{\multicolumn{2}{|c|}{$\begin{array}{l}\text { Veridical } \\
\text { memories }\end{array}$}} & \multirow{2}{*}{\multicolumn{2}{|c|}{$\begin{array}{c}\text { False } \\
\text { memories }\end{array}$}} \\
\hline & & & & & & & & \\
\hline Category & $M$ & $S D$ & $M$ & $S D$ & $M$ & $S D$ & $M$ & $S D$ \\
\hline Self & 77.33 & 20.28 & 80.37 & 22.48 & 58.96 & 20.66 & 56.67 & 32.91 \\
\hline Other & $40.22^{* *}$ & 25.52 & $47.41^{*}$ & 29.15 & 15.48 & 14.43 & $21.11^{*}$ & 24.39 \\
\hline None & 33.04 & 25.64 & 36.67 & 28.48 & 13.70 & 16.69 & 12.59 & 22.08 \\
\hline
\end{tabular}

Note: Data indicate mean percentages $(M)$ and standard deviations $(S D)$ for all "Yes" answers in the recognition memory test (left panel) and for only those "Yes" answers with additional indication of a "remember" experience (right panel), shown for actually presented words (veridical memories) and for critical lures (false memories) separately for the three word categories (Self, Other, None). All "Self" means differ significantly from the corresponding means in the other two categories (all $p s<.001$ ). Critical statistical comparisons refer to differences between the two categories "Other" and "None", in which words were not relevant to the own task and therefore only incidentally encoded: ${ }^{*} p<.05,{ }^{* *} p<.01$, for difference between "Other" and "None". 


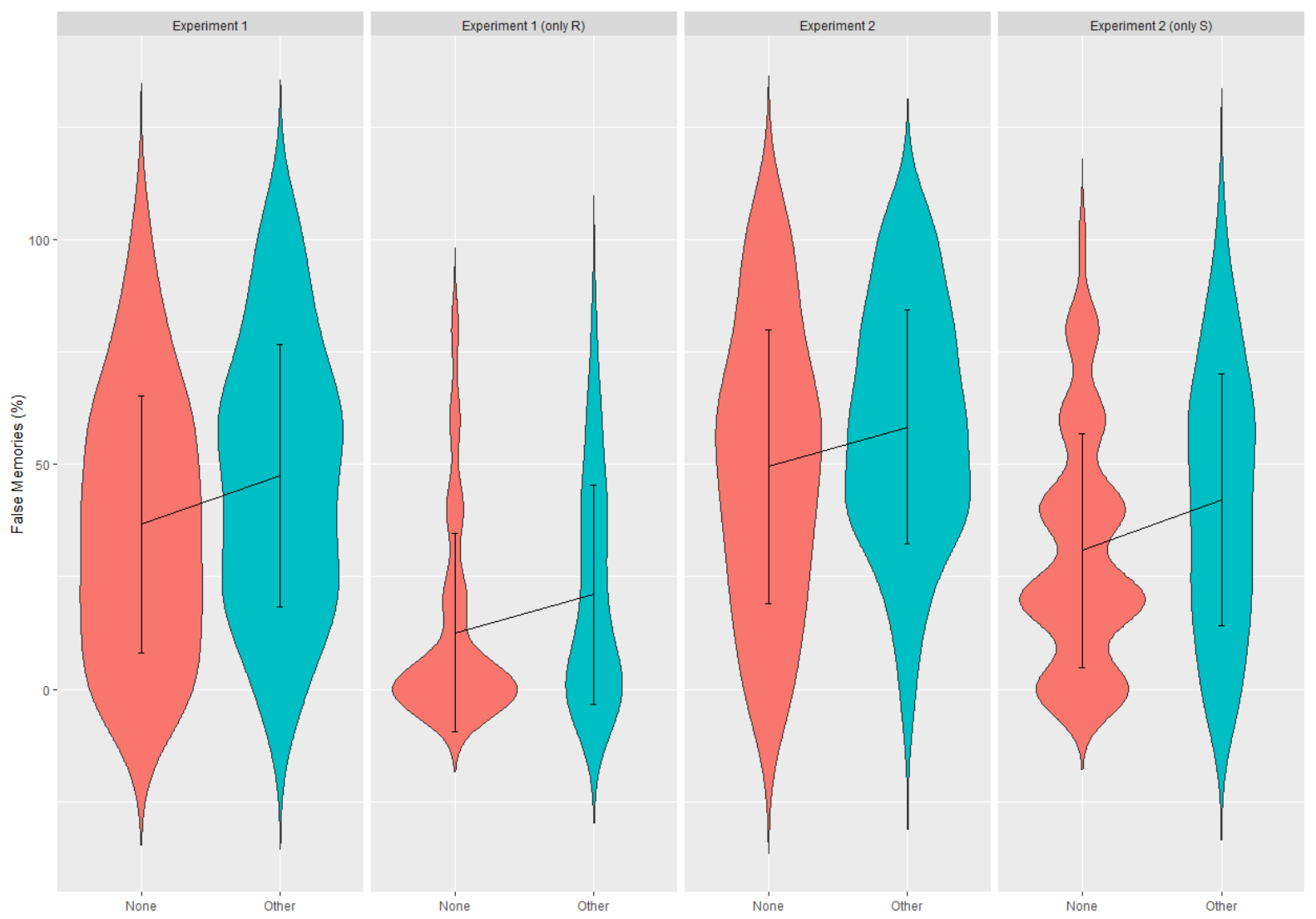

Figure 1. False memories in the two word categories that were not assigned to the self. More false memories were created for the word category assigned to the partner (Other, blue) than for the non-assigned word category (None, red). This was also the case when only subjectively particularly rich memories were considered both in Experiment $1(\mathrm{R}=$ memories including "remember" experiences) and in Experiment 2 ( $\mathrm{S}=$ memories including source memory). Inlays in distribution plots indicate means and standard deviations. 